CASE REPORT

\title{
Coronary-Pulmonary Arterial Fistula in a Heart Transplant Patient
}

\author{
Weiliang Huang, MBBS, MRCP*D
}

Associate Consultant, Department of Cardiology, Changi General Hospital, Singapore

*Corresponding author: Weiliang Huang, MBBS, MRCP, Associate Consultant, Department of Cardiology, Changi General Hospital, 2 Simei Street 3, 529889, Singapore

\section{Introduction}

A coronary arterial fistula (CAF) is a connection between a coronary artery and a cardiac chamber or another blood vessel. Coronary artery fistulae are rare, with a reported incidence of $0.2 \%$, and primarily congenital [1]. However, they have also been reported after cardiac surgery $[2,3]$.

It has been reported that the incidence of CAF in post-surgical individuals seems to rise with the increasing complexity of the surgery. Heart transplant sees the

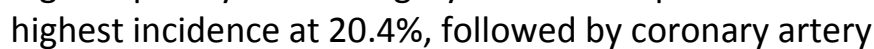
bypass ( $C A B G$ ) with valve surgery, then $C A B G$ alone [4]. While the exact mechanism is unknown, a possible cause could be endothelial and vascular growth factor activation from injury.

The follow up of heart transplant patients is also implicated in the formation of CAF. Most of the post-transplant CAF are cameral-coronary fistulae, and the right ventricle is the most commonly-involved chamber. Heart transplant patients get regular cardiac biopsies to look for rejection, especially in the period just after transplant. It is believed that repeated right ventricular biopsy is associated with the formation of CAF. The incidence of cameral-CAF is noted to have declined over the years in cardiac transplant patients with the development of better endomyocardial biopsy techniques, such as using high-resolution fluoroscopy, dedicated labs staffed by cardiologists for biopsy, and obtaining specimens from the interventricular septum $[5,6]$.

However, in a pediatric group of cardiac transplant patients, non-cameral CAF was more common and reported to be predominantly into the ipsilateral recipient pulmonary vasculature. This incidence seemed to correlate with longer donor ischaemic time [7].

Most post-transplant CAF are asymptomatic. However, CAF are known to be complicated by a myriad of conditions, such as myocardial ischaemia, myocardial infarction, congestive cardiac failure, or sudden death [8].

We describe a case of coronary artery-pulmonary artery fistula in a patient who had an orthotopic heart transplant.

\section{Case Report}

A 40-year-old Indian gentleman has a history of dilated cardiomyopathy secondary to viral myocarditis. He was treated with heart failure medications, received an automated implantable cardioverter-defibrillator in 2005, and left ventricular assist device (LVAD) in 2009. He subsequently underwent an orthotopic heart transplant in 2011 at the age of 34 . The donor was a 38-year old male patient with a brain tumour, which was complicated by seizures that resulted in hypoxic-ischaemic encephalopathy. The transplanted heart was noted to have a LV ejection fraction of $50 \%$ with anterior wall hypokinesia and normal coronary arteries. The immediate post-operative period was complicated by arrhythmias (atrial fibrillation, atrial flutter, and supraventricular tachycardia), ischaemic hepatitis, acute kidney injury, sepsis from an unknown source, and metabolic encephalopathy, all of which gradually resolved. The patient was discharged well.

He was followed up closely with endomyocardial biopsies at 1, 3, 4, 6, 8, 10, 16, 21, 26, 31, and 41 weeks post-transplant, and then at $1,2,3$, and 5 years posttransplant. These showed either Grade 1R rejection or

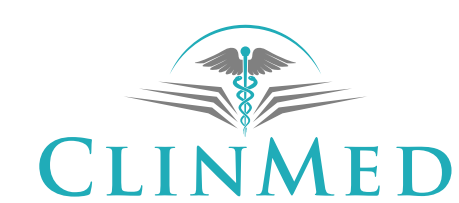

INTERNATIONAL LIBRARY

Citation: Huang W (2020) Coronary-Pulmonary Arterial Fistula in a Heart Transplant Patient. Int J Clin Cardiol 7:172. doi.org/10.23937/2378-2951/1410172

Accepted: April 18, 2020; Published: April 21, 2020

Copyright: (C) 2020 Huang W. This is an open-access article distributed under the terms of the Creative Commons Attribution License, which permits unrestricted use, distribution, and reproduction in any medium, provided the original author and source are credited. 


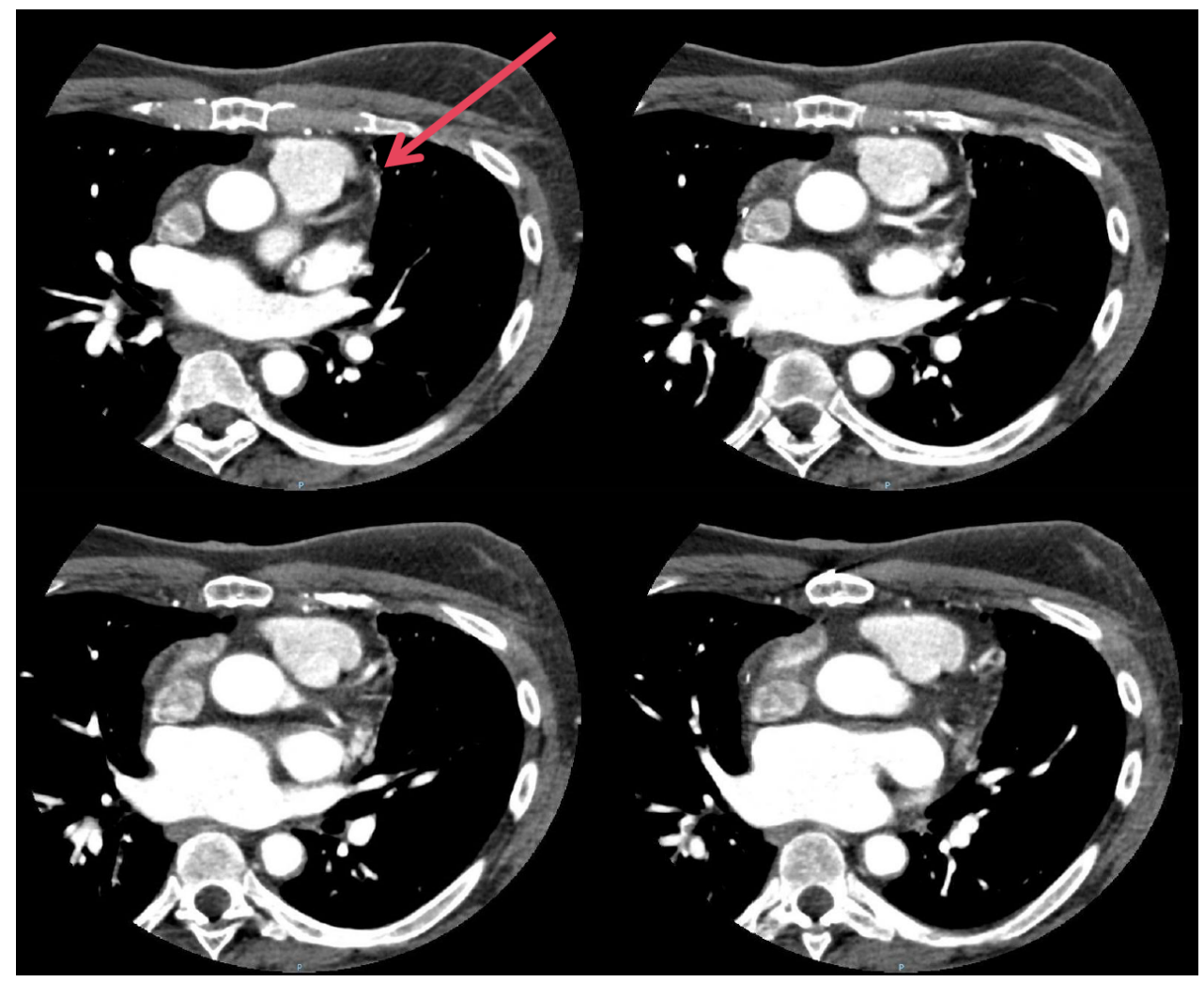

Figure 1: The coronary artery-pulmonary artery fistulae (arrowed) seen on CT coronary angiogram.

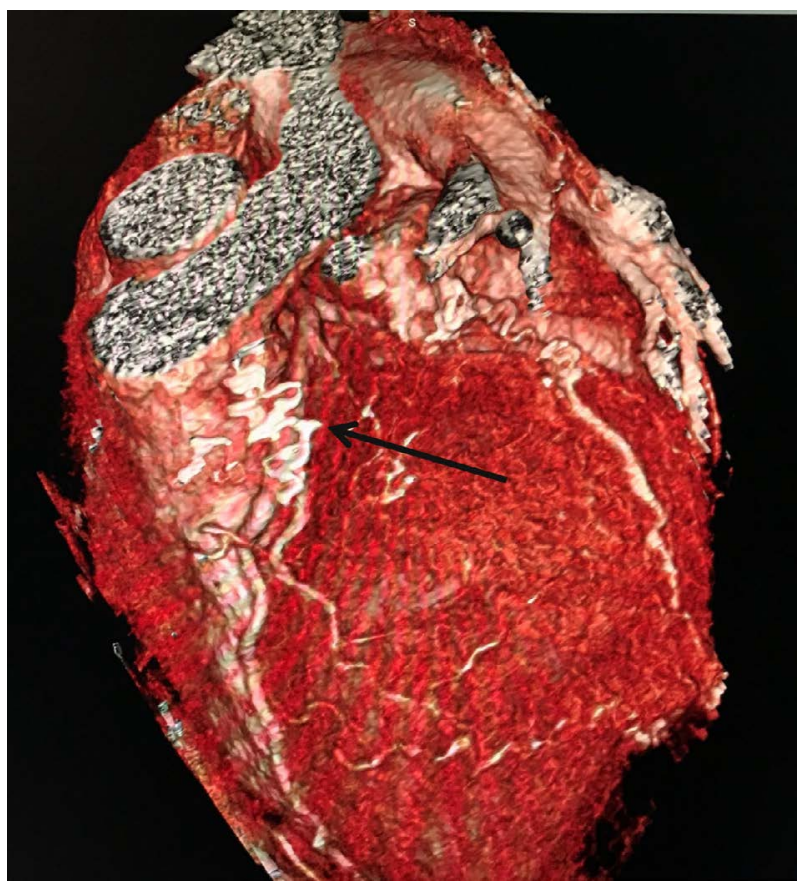

Figure 2: A 3D-reconstruction of the CT coronary angiogram showing the coronary artery-pulmonary artery fistulae (arrowed).

no rejection. Myocardial perfusion imaging done at 2, 3 and 4 years post-transplant was negative for myocardial ischaemia. He also underwent coronary angiograms at 1,3 and 5 years post-transplant, and they showed normal coronary arteries and no fistulae were noted. Serial echocardiograms all showed the preservation of a normal ejection fraction and normal pulmonary artery pressures. A dobutamine-stress echocardiogram performed 5 years after transplantation was negative for myocardial ischaemia.

A dobutamine-stress echocardiogram done 6 years post-transplant, however, showed inducible wall motion abnormality suggestive of myocardial ischaemia in the anterior septal wall. Left and right ventricular size and function were otherwise normal. The left atrium was moderately dilated and the right atrium mildly dilated. Pulmonary artery systolic pressure was estimated to be normal.

A CT coronary angiogram (Figure 1 and Figure 2) showed no significant luminal disease in all three coronary arteries; however, the presence of small vessels originating from the left anterior descending (LAD) artery which extended to the pulmonary trunk, suggestive of small fistulae extending from the coronary artery to the pulmonary artery.

The patient was otherwise asymptomatic and there were no murmurs heard on auscultation. The patient opted not for invasive coronary evaluation at this stage due to the lack of symptoms and the CAF being small on the CT coronary angiogram. He remains on regular follow-up and surveillance.

\section{Discussion}

CAF are primarily congenital and rare. However, an unusually high incidence of CAF has been reported in heart transplant patients.

These are primarily cameral-CAF (to the right ven- 
tricle), possibly as a complication of repeat routine endomyocardial biopsies. Potential causes could involve septal branches into the right ventricle which are 'unroofed' during the performance of right ventricular biopsies, and perhaps also further induced by cytokines (such as endothelial and vascular growth factor) and inflammation from the extensive surgery of cardiac transplantation. It is also possible that in the donor heart these CAF were congenital, or that the donor heart had sustained an injury from an accident prior to harvest and this could further explain non-cameral CAF [9].

Our patient has had a relatively smooth post-transplantation course with minimal rejection, normal coronary arteries, no symptoms, and no related admissions. While he was asymptomatic, his dobutamine-stress echocardiogram was found to be abnormal in the LAD territory, corresponding to the area that might have been affected by the coronary artery-pulmonary artery fistulae [10]. A 'steal' syndrome with substantial blood flowing into the fistula could potentially cause myocardial ischaemia. However, the vessels in question, were noted to be small, and the sizes of the coronary artery and pulmonary artery were normal.

Other known complications of coronary artery fistulae include infective endocarditis and embolic phenomena.

There is limited long-term data on coronary artery fistulae after cardiac transplantation, but outcomes are generally good with conservative management for asymptomatic patients [11]. There are even fewer case reports on fistulae that subsequently underwent intervention. Zoghi, et al. reported an asymptomatic case of coronary-pulmonary arterial fistula that had regional wall motion abnormalities on echocardiography with ischaemia diagnosed on a myocardial perfusion study. The abnormalities resolved with coil embolization of the fistula [12].

As our patient was asymptomatic, the decision was made to continue surveillance for further potential complications.

\section{Conclusion}

Acquired CAF are rare, but have been noted to oc- cur more frequently after cardiac transplant. These patients need to be monitored for potential complications, and treated when necessary. The underlying mechanism of occurrence and incidence should be further explored.

\section{References}

1. Baltaxe HA, Wixson D (1977) The incidence of congenital anomalies of the coronary arteries in the adult population. Radiology 122: 47-52.

2. Shakeel A Qureshi (2006) Coronary arterial fistulas. Orphanet J Rare Dis 1: 51.

3. Somers JM, Verney GI (1991) Coronary cameral fistulae following heart transplantation. Clin Radiol 44: 419-421.

4. Young A, Cheng R, Wei J, Esmailian F, Currier J, et al. (2015) Prevalence of coronary artery fistulae after cardiac surgery. Comparison between coronary artery bypass grafting, valve surgery, and orthotopic heart transplantation. Herz 40: 51-55.

5. Jason M Lazar, Barry F Uretsky (1996) Coronary artery fistula after heart transplantation: A disappearing entity? Cathet Cardiovasc Diagn 37: 10-13.

6. Henzlova MJ, Nath H, Bucy RP, Bourge RC, Kirklin JK, et al. (1989) Coronary artery to the right ventricle fistula in heart transplant recipients: A complication of endomyocardial biopsy. J Am Coll Cardiol 14: 258-261.

7. Allen KY, Goldstein BH, Pahl E, Schumacher KR, Gajarski RJ, et al. (2012) Non-cameral coronary artery fistulae after pediatric cardiac transplantation: A multicenter study. J Heart Lung Transplant 13: 744-749.

8. Kugelmass AD, Manning WJ, Piana RN, Weintraub RM, Baim DS, et al. (1992) Coronary arteriovenous fistula presenting as congestive heart failure. Cathet Cardiovasc Diagn 26: 19-25.

9. Vermeulen T, Haine S, Paelinck BP, Rodrigus IE, Vrints CJ, et al. (2010) Coronary artery-pulmonary artery fistula in a heart-transplanted patient. Eur J Echocardiogr 11: 80-81.

10. Thomas N James, George E Burch (1958) Blood supply of the human interventricular septum. Circulation 17: 391-396.

11. Wei J, Azarbal B, Singh S, Rafiei M, Cheng R, et al. (2013) Frequency of coronary artery fistulae is increased after orthotopic heart transplantation. J Heart Lung Transplant 32: 744-746.

12. Zoghi M, Cınar C, Kurşun M, Nalbantgil S (2015) Coil embolization of iatrogenic coronary-pulmonary arterial fistula after heart transplantation. Anatol J Cardiol 15: 587-588. 